\title{
Agresividad en menores de 18 años jugadores de fútbol: Diferencias en función del sexo y la edad y en comparación con los jugadores de baloncesto
}

\author{
Aggression in under 18 soccer players: Differences in by \\ sex and age and compared to basketball players
}

\section{Agresividade com menos de 18 jogadores de futebol: Diferenças em por sexo e idade e comparados com os jogadores de basquete}

\author{
Mónica Blasco y Mireia Orgilés \\ Universidad Miguel Hernández de Elche
}

\begin{abstract}
Resumen: A pesar de su frecuencia, son escasos los estudios que examinan la conducta agresiva infanto-juvenil en relación al deporte. El objetivo de este estudio piloto es analizar las diferencias en conductas agresivas en menores de 18 ańos jugadores de fútbol en función del sexo y la edad, y en comparación con los jugadores de baloncesto. Participaron 316 menores con edades comprendidas entre 7 y 17 años. Se administró, al finalizar uno de los partidos disputados, la Escala de Agresión Física y Verbal (AFV, Caprara y Pastorelli, 1993), así como un cuestionario sociodemográfico. Se hallaron diferencias significativas $(p<.05)$ en función del sexo en la práctica de fútbol, de modo que las mujeres mostraron mayor agresividad física que los hombres. Respecto a la edad, los menores de 13 a 17 años jugadores tanto de fútbol como de baloncesto mostraron mayor agresividad verbal que los menores de 7 a 12 ańos ( $p<.05, p<.01$ respectivamente). Los resultados obtenidos indican que no existen diferencias en la agresividad presentada entre los jugadores de ambos deportes. Se discuten los hallazgos del estudio, que aporta una nueva visión acerca de la agresividad en el deporte de los menores de 18 ańos.

Palabras clave: conducta, agresiva, menores, fútbol, baloncesto

Abstract: Despite its frequency, there are few studies examining the child and adolescent aggressive behavior in relation to sport. The aim of this pilot study is to analyze the differences in aggressive behavior in under 18 soccer players according to sex and age, and compared to basketball players . Participants 316 children aged between 7 and 17 years. Was administered at the end of one of the games, the Physical Aggression Scale and Verbal ( AFV, Caprara and Pastorelli, 1993 ) and a sociodemographic questionnaire.
\end{abstract}

Significant differences $(p<.05)$ by sex in soccer practice, so is found that women showed more physical aggression than men. Regarding age, the under 13 and 17 both football players and basketball showed more verbal aggression than children 7 to 12 years $(p<.05, p<.01$ respectively). The results indicate that there are no differences in aggression displayed between the players of both sports. The findings of the study are discussed, which provides a new point of view of aggression in children and adolescents. Key words: behavior, aggressive, children, football, basketball.

Resumo: Apesar de freqüente, há poucos estudos que examinam a criança eo comportamento agressivo de adolescentes em relação ao esporte . O objetivo deste estudo piloto é analisar as diferenças de comportamento agressivo em menos de 18 jogadores de futebol de acordo com sexo e idade, e em comparaçáo com os jogadores de basquete. Os participantes 316 crianças com idade entre 7 e 17 anos. Foi administrada no final de um dos jogos , a Escala de agressão física e verbal ( AFV , Caprara e Pastorelli , 1993) e um questionário sociodemográfico. Diferenças significativas $(\mathrm{p}<0,05)$ por sexo na prática do futebol, para que as mulheres mostraram agressão física mais do que os homens foram encontrados. Quanto à idade, os menores de 13 e 17 jogadores no futebol e basquete mostrou mais agressividade verbal do que as crianças de 7-12 anos ( $\mathrm{p}<0,05, \mathrm{p}<0,01$, respectivamente ). Os resultados indicam que não existem diferenças de agressão exibidos entre os jogadores de ambos os esportes. Os resultados do estudo, o que traz uma nova visão de agressão no esporte de crianças menores de 18 anos são discutidas.

Palavras-chave: comportamento, crianças agressivas, futebol, basquetebol

En la actualidad, los comportamientos desadaptativos relacionados con la práctica del deporte que se dan en los centros escolares son cada vez más frecuentes, preocupando tanto a profesionales del ámbito escolar como a los propios padres. A pesar de que el deporte es una alternativa educativa muy

Dirección para correspondencia [Correspodence address]: Mireia Orgilés. Universidad Miguel Hernández de Elche, Departamento de Psicología de la Salud, Avda. de la Universidad s/n, 03202 Elche, Alicante, España; telf 96665 8348; E-mail: morgiles@umh.es beneficiosa para los menores, se dan actuaciones impropias por parte de jugadores, espectadores y entrenadores con una incidencia excesiva (Cox, 2008). Las conductas agresivas han llegado a ser demasiado habitual en las gradas, en los banquillos y sobre todo en el terreno de juego. Estos actos agresivos en el terreno deportivo son calificados de inadecuados por algunos deportistas, pero al mismo tiempo los consideran convenientes (Bredemeier y Shields, 1986). Algunos autores indican que los comportamientos agresivos facilitan los resultados en cuanto al rendimiento (Widmeyer y Birch, 1984), 
mientras que otros (Silva, 1980) afirman lo contrario y defienden que el aumento del nivel de arousal desvía la atención del jugador hacia aspectos no relacionados con la ejecución, como es lesionar al contrario.

Tradicionalmente, la agresión se ha definido como un comportamiento que va dirigido a dańar a alguien (Dollard, Doob, Miller, Mowrer y Sears, 1939), por lo que conlleva intencionalidad. Bandura (1987), sin embargo, no habla de intención al definir la agresión como una conducta que produce un daño personal y una destrucción física. El término de agresión psicológica se introdujo con posterioridad, cuando Berkowitz (1993) define la agresión como cualquier conducta que pretende herir física o psicológicamente a alguien. Husman y Silva (1984) diferencian entre agresión hostil, que es la que se lleva a cabo con el objetivo de causar daño, y agresión instrumental, cuyo objetivo es conseguir un incentivo. Tanto una como otra causan dańo y ninguna es aceptable.

La mayoría de autores explican la agresión mediante la teoría del aprendizaje social. El mayor defensor de esta teoría fue Bandura (1987), quien afirma que la agresión es una conducta aprendida mediante la observación y el modelado de las conductas de los otros, seguida de la obtención de refuerzos por actuar de forma similar. Smith (1988) observó que los jugadores jóvenes de hockey sobre hielo imitaban la violencia que se produce en el juego profesional. Además, percibían en la televisión cómo sus héroes modelaban los comportamientos agresivos y recibían reconocimiento personal por exhibir una conducta parecida. Las investigaciones sobre el aprendizaje social en el deporte muestran que a la mayor parte de los deportistas no se les ha enseńado a ser violentos de una manera explícita, pero los actos agresivos pueden aparecer de forma sutil y se da en todos los deportes. Esta teoría cuenta con un gran respaldo científico y hace hincapié en la influencia de las personas significativas sobre el desarrollo de la agresión (Cox, 2008).

Diversos trabajos han examinado la conducta agresiva en función del sexo y de la edad, pero se carece de estudios que evalúen dichos comportamientos de forma específica en menores deportistas. Con población general, Sandoval (2001) llevó a cabo una investigación en la que comprobó que los hombres son más agresivos que las mujeres y éstas son más prosociales que los hombres. Con su estudio se evidencia cómo la escuela y la interacción tanto con los iguales como con las figuras de autoridad influye en el desarrollo de comportamientos sociales. Lobato (2005) concluyó, a través de un sociograma, que las diferencias de sexo se presentan desde edades muy tempranas, observando que las mujeres dirigen sus juegos hacia las relaciones interpersonales, el cuidado y las tareas domésticas mientras que los niños se centran en juegos de acción. Alandette y Hoyos (2009) confirmaron que existen diferencias en función del sexo en la conducta agresiva, siendo los adolescentes varones los que alcanzan las pun- tuaciones más altas respecto a las mujeres de su misma edad. Por otro lado, West y Farrington (1973) realizaron un estudio longitudinal en el que confirmaron que el progreso de la edad está relacionado positivamente con el aumento de las conductas agresivas. Robins (1986) demostró que el número de trastornos de conducta presentados en la infancia predice el número de conductas antisociales en la edad adulta, siendo el más significativo el problema de la agresión. Las investigaciones realizadas por Olweu (1979) y Farrington (1991) llevadas a cabo en el ámbito de la conducta agresiva en niños y adolescentes señalan que dicha conducta tiende a estabilizarse con el tiempo, pero sobre todo a aumentar hacia comportamientos más complejos como por ejemplo la delincuencia. En la misma línea, Lochman et al. (1993), encontraron una alta correlación entre la conducta agresiva de los niños en edad escolar y la conducta antisocial de la adolescencia y la adultez.

En diferentes estudios relacionados con el fútbol se ha comprobado que en la gran mayoría de partidos de fútbol base se llevan a cabo conductas agresivas y violentas que no están permitidas en el reglamento. Ejemplo de ello es el estudio realizado por Cervelló y García Calvo (2003) en el que se muestra cómo los árbitros sancionaron más de 300 conductas agresivas durante un campeonato de liga con jugadores de 11, 12 y 13 años. En su investigación sobre la conducta agresiva en los partidos de fútbol, Volkamer (1971) observó que la frustración era la causa de un mayor número de faltas del equipo perdedor. Además, también confirmó que las infracciones forman una gran parte de las estrategias llevadas a cabo en el fútbol (Lefebre y Passer, 1974; Volkamer, 1971; Widmeyer y Birch, 1984). Según Frogner y Pilz (1982), las conductas agresivas en el deporte aumentan con la edad al convertirse en una conducta aceptada socialmente. Además, el significado de "juego limpio" parece hacerse menos importante con la edad para tratar de conseguir que el equipo gane con la mayor ventaja posible (Heinilä, 1974). Pfister y Sabatier (1994) afirman que el número de agresiones aumentaba en los partidos cuanta mayor era la edad y la experiencia. Además, dichos autores destacan la variable sexo como predictora de la agresión, siendo los niños más agresivos que las nińas (Pelegrín, 2001).

La mayoría de los estudios sobre el comportamiento agresivo en el fútbol se han llevado a cabo sin comparar la conducta agresiva de sus jugadores con la de jugadores de otros deportes. El objetivo del presente estudio es por tanto analizar las diferencias en el comportamiento agresivo en función del sexo y la edad en una muestra de menores de 18 años jugadores de fútbol, y compararles con los que juegan a baloncesto. Se ha seleccionado el baloncesto como deporte de comparación debido a las características que comparte con el fútbol; ambos son deportes de equipo, se juegan en el mismo medio, son los deportes con más relevancia en los medios de comunicación, y son los que practican con más frecuencia los 
menores. En base a este objetivo, se plantean las siguientes hipótesis (a) Tanto los jugadores de fútbol como los de baloncesto presentarán mayor agresividad que las jugadoras, (b) Tanto los menores de 13 a 17 ańos que juegan al fútbol como los que juegan al baloncesto presentarán mayor agresividad que los menores de 7 a 12 años, y (c) Los menores que juegan al fútbol presentarán mayor agresividad al finalizar el partido que los que juegan al baloncesto.

\section{Método}

\section{Participantes}

En este estudio se reclutaron un total de 316 menores pertenecientes a distintos clubs de fútbol y baloncesto de la provincia de Alicante con edades comprendidas entre los 7 y 17 años de edad. El 47.15\% ( $n=149)$ eran jugadores de fútbol y el $52.85 \%(n=167)$ de baloncesto, con una edad media de $12.55(D T=2.33)$ y $12.19(D T=2.72)$ respectivamente. En la tabla 1 se presenta el número de participantes y su porcentaje en función de la edad y el sexo en ambas submuestras.

Tabla 1. Características de la muestra

\begin{tabular}{|c|c|c|c|c|c|c|}
\hline \multirow{3}{*}{ Jugadores } & \multicolumn{4}{|c|}{ Sexo } & \multicolumn{2}{|c|}{ Edad } \\
\hline & \multicolumn{2}{|c|}{ Mujeres } & \multicolumn{2}{|c|}{ Hombres } & \multirow[t]{2}{*}{$M$} & \multirow[t]{2}{*}{$D T$} \\
\hline & $\%$ & $n$ & $\%$ & $n$ & & \\
\hline Fútbol $(n=149)$ & 50.33 & 76 & 44.24 & 73 & 12.55 & 2.33 \\
\hline Baloncesto $(n=167)$ & 49.67 & 75 & 55.76 & 92 & 12.19 & 2.73 \\
\hline Total $(n=316)$ & 47.78 & 151 & 52.22 & 165 & 12.36 & 2.55 \\
\hline
\end{tabular}

\section{Instrumentos de evaluación}

El instrumento aplicado para evaluar la agresividad fue la adaptación española de la Escala de Agresión Física y Verbal (AFV; Caprara y Pastorelli, 1993) de del Barrio, Moreno y López (2001). Consta de 20 ítems referentes a conductas agresivas tanto físicas como verbales, por ejemplo "tengo ganas de pegar" o "insulto a los compañeros". La puntuación se registra con una escala tipo Likert donde $1=$ nunca, $2=$ algunas veces y 3 = a menudo. Incluye cuatro ítems de control para evitar patrones de respuesta pero que no se puntúan. El punto de corte para la versión española es de 31 y 32 para la agresión física y verbal, respectivamente. La versión española ha demostrado un alfa de Cronbach que ha oscilado de 0.84 (del Barrio, et al., 2001).

\section{Procedimiento}

La muestra para este estudio piloto se seleccionó por conveniencia de cuatro clubs de fútbol y baloncesto de la provincia de Alicante. Se precisó de autorizaciones por parte del equipo directivo, de los entrenadores y, al ser los participantes menores de edad, el consentimiento informado de los padres de los sujetos. Los cuestionarios fueron aplicados después de un partido de liga por los entrenadores, quienes habían recibido información y preparación para ello.

\section{Resultados}

Se comprobó la normalidad de la muestra mediante la prueba de Kolmogorov-Smirnov. Se obtuvo un valor de $p=.000$ en todas las variables incluidas en el estudio, indicando que la muestra no era normal, por lo que se utilizó la prueba $U$ de Mann-Whitney para muestras independientes con el objetivo de conocer si existían diferencias en las puntuaciones en agresión verbal y física en función del sexo y la edad, así como entre los jugadores de ambos deportes. Para conocer la magnitud de las diferencias se halló el tamaño del efecto de Cohen de las comparaciones que resultaron significativas, considerándose 0.20 un tamaño pequeño, 0.50 medio y 0.80 grande (Cohen, 1988).

Se evaluaron las diferencias en agresividad física y verbal entre los jugadores de fútbol hombres y mujeres (Tabla 2). Los resultados muestran diferencias significativas únicamente en agresividad física, con un tamaño del efecto pequeño $(d=0.35)$, presentando las chicas mayores puntuaciones en agresividad que los chicos. En los jugadores de baloncesto no se hallaron diferencias estadísticamente significativas entre chicos y chicas en ninguno de los tipos de agresividad estudiados $(p>.05)$ (Tabla 2). 
Tabla 2. Diferencias en agresividad física, verbal y total entre jugadores y jugadoras de fútbol y baloncesto

\begin{tabular}{|c|c|c|c|c|c|c|}
\hline \multirow[t]{2}{*}{ Jugadores } & & \multicolumn{2}{|c|}{$\begin{array}{c}\text { Hombres } \\
(n=73)\end{array}$} & \multicolumn{2}{|c|}{$\begin{array}{l}\text { Mujeres } \\
(n=76)\end{array}$} & \multirow[t]{2}{*}{$U$} \\
\hline & & $M$ & $D T$ & $M$ & $D T$ & \\
\hline \multirow[t]{2}{*}{ Fútbol } & Agresividad física & 10.23 & 2.14 & 11.07 & 2.56 & $2247^{*}$ \\
\hline & Agresividad verbal & 12.79 & 2.25 & 13.32 & 2.53 & 2534.5 \\
\hline \multirow[t]{2}{*}{ Baloncesto } & Agresividad física & 10.54 & 3.12 & 10.35 & 2.65 & 3230 \\
\hline & Agresividad verbal & 12.98 & 3.36 & 12.69 & 2.67 & 2517.5 \\
\hline
\end{tabular}

Respecto a la edad, en la submuestra que practicaba fútbol, fueron significativas las diferencias en agresividad verbal con un tamaño del efecto pequeño $(d=0.33)$. Entre los jugadores de baloncesto, también se hallaron diferencias significativas en la agresividad verbal, siendo también pequeño el tamaño del efecto $(d=0.30)$. En ambos casos, las puntuaciones en agresividad verbal son mayores en los menores de 13 a 17 años que en los menores de 7 a 12 años (Tabla 3).

Tabla 3. Diferencias en agresividad física, verbal y total entre menores de 7 a 12 años y menores de 13 a 17 años jugadores de fútbol y baloncesto

\begin{tabular}{llccccc}
\hline Jugadores & & \multicolumn{2}{c}{$\begin{array}{c}\text { Menores de } \\
7 \text { a } 12 \text { años } \\
(n=74)\end{array}$} & \multicolumn{2}{c}{$\begin{array}{c}\text { Menores de } \\
13 \text { a 17 años } \\
(n=75)\end{array}$} & $U$ \\
\cline { 3 - 6 } & & $M$ & $D T$ & $M$ & $D T$ & \\
\hline \multirow{2}{*}{ Fútbol } & Agresividad física & 10.53 & 2.37 & 10.79 & 2.43 & 2585.5 \\
& Agresividad verbal & 12.66 & 2.43 & 13.45 & 2.32 & $2258^{*}$ \\
Baloncesto & Agresividad física & 10.40 & 3.08 & 10.51 & 2.76 & 3230 \\
& Agresividad verbal & 12.39 & 3.42 & 13.31 & 2.60 & $2517.5^{* *}$ \\
\hline
\end{tabular}

${ }^{*} p<.05,{ }^{* *} p<.01$

Por último, no se hallaron diferencias estadísticamente significativas ni en agresividad física ni en agresividad verbal entre los jugadores de fútbol y los de baloncesto ( $\mathrm{p}>.05)$, evaluada en ambos casos tras disputar un partido (Tabla 4).

Tabla 4. Diferencias en agresividad física y verbal entre deportes

\begin{tabular}{lccccc}
\hline & \multicolumn{2}{c}{$\begin{array}{c}\text { Fútbol } \\
(n=149)\end{array}$} & \multicolumn{2}{c}{$\begin{array}{c}\text { Baloncesto } \\
(n=167)\end{array}$} & $U$ \\
\cline { 2 - 5 } & $M$ & $D T$ & $M$ & $D T$ & \\
\hline Agresividad física & 10.61 & 2.35 & 10.46 & 2.91 & 11102.5 \\
Agresividad verbal & 13.01 & 2.36 & 12.85 & 3.06 & 11333 \\
\hline
\end{tabular}

\section{Discusión}

El objetivo de este estudio era examinar las diferencias en agresividad física y verbal entre los menores de 18 años jugadores de fútbol y baloncesto, así como conocer si existían diferencias en la conducta agresiva en función del sexo y de la edad. Los resultados muestran que las diferencias por sexo son significativas en la agresividad física entre los hombres y mujeres que jue- gan al fútbol, pero no se aprecian diferencias en los que juegan al baloncesto. En contra de lo esperado, y aunque el tamaño del efecto es pequeño, las mujeres muestran puntuaciones más elevadas en agresividad física que los hombres. Los resultados de nuestro estudio no confirman por tanto los hallazgos de estudios previos con población general que indican que los hombres son más agresivos que las mujeres (Alandette y Hoyos, 2009; Pelegrín, 2001; Sandoval, 2001). Aunque la razón por la 
que las mujeres se muestran más agresivas que los hombres en la práctica de fútbol no está clara, es posible que las dificultades que tradicionalmente la sociedad ha impuesto a las mujeres para ser reconocidas como jugadoras de fútbol pueda influir en que se comporten de forma más agresiva que los hombres en el ámbito del deporte futbolístico. Quizás su intento de demostrar su valía en el terreno de juego les lleve a una práctica agresiva del deporte. No obstante, es necesario llevar a cabo nuevos estudios para poder confirmar dicha propuesta explicativa. Puesto que las investigaciones previas llevadas a cabo sobre diferencias en la conducta agresiva en función del género se han realizado mayoritariamente en el contexto escolar y no en el ámbito deportivo, no es posible comparar los resultados de nuestra investigación con otros hallazgos. Respecto a la edad y de acuerdo a lo esperado, los menores de 13 a 17 ańos que juegan tanto al fútbol como al baloncesto presentan mayor agresividad verbal que los menores de 7 a 12 ańos, aunque sus puntuaciones en agresividad física no difieren. Estos resultados coinciden con los obtenidos por Frogner y Pilz (1982), que defendían que a mayor edad mayor agresividad en el deporte, y con los de Pfister y Sabatier (1994), quienes afirmaban que existía una correlación directa entre el número de agresiones físicas en los partidos y la edad de los sujetos. Este estudio, a pesar de que únicamente halló diferencias significativas en la agresividad verbal y no física, es acorde a dicho hallazgo, señalando que la agresividad verbal en los jugadores es mayor en la adolescencia que en la infancia. La ausencia de diferencias significativas entre los menores de 7 a 12 años y los menores de 13 a 17 años en la agresividad física autopercibida después del partido quizás sea debida a las sanciones que conlleva tener conductas agresivas físicas en el terreno de juego. Evitar dichas sanciones quizás podría motivar al adolescente a controlar su agresividad física, pero no la agresividad verbal que es más sutil y menos perceptible.

A diferencia de lo planteado en nuestra hipótesis, no se hallaron diferencias significativas en el nivel de agresividad entre los jugadores de fútbol y de baloncesto al finalizar un partido. La ausencia de diferencias entre los jugadores de ambos deportes podría ser debido a que las conductas agresivas no se ven influidas por los medios de comunicación, en los que el fútbol tiene un protagonismo mayor. Además, ambos son los deportes más practicados en el ámbito escolar, por lo que es posible que los menores que juegan al fútbol y baloncesto en competiciones organizadas hayan recibido un aprendizaje similar respecto a las exigencias, competitividad, y normas de comportamiento en el campo entre las que se encuentra la evitación de conductas agresivas, pudiendo suponer una si- militud, a nivel general, en la manera de practicar el deporte. La supervisión del juego por parte de entrenadores y padres puede también influir en que los menores, a diferencia de los adultos, no se comporten de forma tan agresiva al disputar un partido. Por tanto, parece que existen determinadas variables que median en el comportamiento de los menores, de modo que la observación de modelos agresivos en el juego profesional de adultos, tal y como indica la teoría del aprendizaje social, no influye directamente en la agresividad de los jóvenes, como se ha observado en otros deportes (Smith, 1988). A pesar de que tradicionalmente el fútbol se ha considerado un deporte en el que la conducta agresiva por parte de sus jugadores es más frecuente que en el baloncesto, no existen estudios previos que analicen dicha conducta en menores, por lo que no es posible comparar nuestros hallazgos. Por otro lado, los participantes de este estudio no superaron el punto de corte establecido para considerar que su conducta es agresiva, por lo cual quizás sería conveniente examinar en investigaciones futuras las diferencias en agresividad en una muestra de jugadores de fútbol y baloncesto que sí muestren un nivel significativo de conductas agresivas.

Entre las limitaciones del estudio, destaca la falta de información sobre determinadas variables que podrían condicionar los resultados del estudio. Entre ellas, si los jugadores han ganado o perdido el partido, el tiempo de juego de cada participante, o si el partido se llevó a cabo en el campo del jugador o en el del equipo contrario. Sería por tanto conveniente desarrollar estudios que examinen de forma específica la conducta agresiva en las menores de edad jugadoras de fútbol, con el objetivo de determinar las variables que pueden estar influyendo en su comportamiento en el campo y por tanto en su nivel de agresividad autopercibida al finalizar una competición. A pesar de las limitaciones señaladas, este trabajo aporta una nueva visión acerca de las conductas agresivas en menores de 18 ańos, y presenta como novedad la comparación de la conducta agresiva entre jugadores de fútbol y baloncesto.

\section{Aplicaciones prácticas}

Las aplicaciones prácticas de este estudio son claras. Se considera importante la promoción de conductas prosociales y de habilidades sociales alternativas a la agresión para prevenir las conductas agresivas tanto físicas como verbales en los equipos deportivos. Del mismo modo, llevar a cabo un seguimiento y una evaluación continua del comportamiento agresivo durante el juego de los más pequeńos podría evitar una escalada en dicha conducta a lo largo de los años.

\section{Referencias}

1. Alandette, Y. y Hoyos, O. (2009). Representaciones mentales sobre los tipos de agresión en escolares. Psicología desde el Caribe, 24, 1-25.

2. Bandura, A. (1987). Teoría del aprendizaje social. Madrid: Espasa-Calpe.
3. Berkowitz, L. (1993). Some determinants of impulsive aggression: the role of mediated association with reinforcement for aggression. Psychological Review, 81, 165-176. 
4. Bredemeier, B. y Shields, D. (1986). Moral growth among athletes and nonathletes: A comparative analysis. Journal of Genetic Psychology, 147, 7-18.

5. Cervelló, E. y García-Calvo, T. (2003). Clima motivacional y patrones de conducta desadaptativos en jóvenes jugadores de fútbol. Manuscrito no publicado.

6. Cohen, J. (1988). Statistical Power Analysis for the Behavioral Sciences. $\left(2^{\text {nd }}\right.$. Eds.), Hillsdale, N.J., Erlbaum.

7. Cox, R.H. (2008). Psicología del deporte: Conceptos y sus aplicaciones. Madrid: Editorial Médica Panamericana S.A.

8. Del Barrio, M. V., López, R. y Moreno, C. (1993). Adaptación de la Escala de Agresión Física y Verbal (AFV; Caprara y Pastorelli, 1993) a población española. En Asociación de Evaluación Psicológica (Eds.), Actas del V Congreso de Evaluación Psicológica (pp. 15-22). Málaga: Universidad de Málaga.

9. Del Barrio, M. V., López, R. y Moreno, C. (2001). Evaluación de la agresión y la inestabilidad emocional en niños españoles: Su relación con la depresión. Clinica y Salud, 12, 33-50.

10. Dollard, J., Doob, L. W., Miller, N. E., Mowrer, O. H., y Sears, R. F. (1939). Frustration and Aggression. New Haven: Yale University Press.

11. Farrington, D.P. (1991). Childhood aggression and adult violence: Early precursors and later life outcomes. En D. J. Pepler y K. H. Rubi (Eds.), The development and treatmen of childhood aggression (pp. 5-29). Hillsdale, NJ: Erlbaum.

12. Frogner, E. y Pilz, G. A. (1982). Untersuchung zur EÏnstellung von jugendlichen Fussballspielern und spielerinnen zu Regeln und Normen im Sport (Investigaciones de las actitudes de jóvenes jugadores y jugadoras de fútbol frente a normas y reglas). En G.Pilz (ed.), Sport und Gemalt (Deporte y violencia). Schorndorf: Verlag Kart Hofmann.

13. Heinilä, K. (1974). Ethics of Sport. Department of Sociology and Planning for Physical Culture, Informe de investigación, 4. University of Jyvaskyla.

14. Husman, B. F. y Silva, J.M. (1984). Aggression in sport: Definitional and theoretical considerations. In Silva, J. M. and Weinberg, R.S. (Eds.). Psychological foundations of sport (pp. 246-260). Champaign, IL, Human Kinetics.

15. Lefebvre, L. y Passer, M. (1974). The effects of game location and im- portant on aggression in team sport. International Journal of Sport Psychology, 5, 102-110.

16. Lobato, E. (2005). Juego sociodramático y esquemas de género. Una investigación en educación infantil. Revista virtual Cultura y educación, 17, 115-129.

17. Lochman, J., Coie, J., Underwood, M., y Terry, R. (1993). Effectiveness of a social relations intervention program for aggressive and nonaggressive rejected children. Journal of Consulting and Clinical Psycholy, 61, 1053-1058.

18. Olweu, S. (1979). Stability of aggressive behavior patterns in make a review. Psychological Bulletin, 86, 852-875.

19. Pelegrín, A. (2001). La participación deportiva en la infancia y la juventud: Desarrollo Psicológico y Social. Encuentro Profesional de Psicología del Deporte: I Jornadas Técnicas, Murcia.

20. Pfister, R. y Sabatier, C. (1994). Aggressive interactions in sports practical young. Enfance, 2-3, 215-232.

21. Robins, L. (1986). Changes in conduct disorders over time. In Farren, D.C y McKinney, J.D. (Eds.). Risk in Intellectual and Psychosocial Development (pp. 227- 259). New York: Academic Press.

22. Sandoval, J. (2001). Ambiente escolar, familiar y comunitario en relación con los comportamientos agresivos y prosociales en niños de 3 a 12 años. Revista Facultad Nacional Salud Pública, 24, 30-39.

23. Silva, J. (1980). Understanding aggressive behavior and its effects upon athletic performance. En W.F Straub (Ed.), Sport psychology: An analysis of athlete behavior in sport (2nd ed., pp. 177-186). Ithaca, NY Mouvement.

24. Smith, M. D. (1988). Interpersonal sources of violence in hochey: The influence of parents, coaches, and teanmates. En F.L. Smoll, R.A. Magill y M.J. Ash (Eds.). Children in sport (3a ed., pp. 301-313), Champaign, IL, Human Kinetics.

25. Volkamer, M. (1971). Aggression oriented social systems rivalry. An investigation into football. Sportwissenschaft, 1, 33-64.

26. West D., y Farrington D. (1973). Who becomes delinquent? Second report of all Cambridge study in delinquent development. London: Heinemann

27. Widmeyer, W. N. y Birch, J. S. (1984). Aggression - performance relationships in sport, en J. M. Silva y R. S. Weinberg (Eds.), Psychological foundations of sport (pp. 274-286), Champaign, IL, Human Kinetics. 